\title{
Eficiencia en la ejecución de proyectos de inversión
}

\author{
Soto Arévalo, Rafael \\ rafo388@ hotmail.com \\ Doctorando Universidad César Vallejo \\ Tarapoto - Perú.
}

\section{RESUMEN}

La eficiencia en la ejecución de proyectos de inversión pública abarca una serie de herramientas que permiten asegurar que las actividades y obras públicas puedan realizarse de manera efectiva en base a ciertos criterios a fin que se pueda mejorar la prestación de bienes y servicios públicos destinados a optimizar la calidad de vida de los ciudadanos. La investigación tuvo un enfoque cualitativo, de tipo básico y diseño de revisión sistémica, estuvo constituida por 10 artículos científicos que formaron parte de la información bibliográfica, por cuanto sólo tales artículos tuvieron un contenido claro y preciso respecto a la eficiencia en la ejecución de proyectos de inversión; posteriormente al análisis de los artículos se llegó a concluir que, con el trascurso de los años, se han ido incrementando las deficiencias e inconvenientes que se presentan en la ejecución de proyectos de inversión, los mismos que imposibilitan la optimización de su eficiencia y origina que los pobladores atraviesen diversas dificultades para satisfacer sus necesidades básicas, frente a ello se pudo reconocer la necesidad de adoptar estrategias que coadyuven en el manejo de los recursos públicos y garanticen el crecimiento y desarrollo del país.

Palabras clave: Eficiencia, ejecución, proyectos de inversión. 


\title{
Efficiency in the execution of investment projects
}

\begin{abstract}
Efficiency in the execution of public investment projects encompasses a series of tools that ensure that public activities and works can be carried out effectively based on certain criteria in order to improve the provision of public goods and services aimed at optimizing the quality of life of citizens. The research had a qualitative approach, of a basic type and design of a systemic review, it consisted of 10 scientific articles that were part of the bibliographic information, since only such articles had a clear and precise content regarding the efficiency in the execution of projects investment; After analyzing the articles, it was concluded that, over the years, the deficiencies and inconveniences that arise in the execution of investment projects have increased, which make it impossible to optimize their efficiency and originate that The inhabitants go through various difficulties in satisfying their basic needs. Faced with this, the need to adopt strategies that contribute to the management of public resources and guarantee the growth and development of the country was recognized.
\end{abstract}

Keywords: Efficiency, execution, investment projects.

Artículo recibido: 15 abril 2021

Aceptado para publicación: 19 abril 2021

Correspondencia: 1montilla@ucv.edu.pe Conflictos de Interés: Ninguna que declarar 


\section{INTRODUCCIÓN}

Los diversos cambios producidos a consecuencia de la globalización afectaron de manera significativa la gestión pública, siendo uno de los estados más afectados el Estado Colombiano, pues se pudo percibir que existe diversos cambios normativos que implican la exigencia en la adecuación de procedimientos y estructuras administrativas eficientes, las mismas que deben ser incorporadas a las dinámicas propias de la modernización de tal manera que pueda optimizar su funcionamiento a través del cumplimiento de los principios de efectividad, economía y celeridad y, a su vez, garantizar la ejecución de proyectos de inversión de manera eficaz (Gonzáles, 2019). Asimismo, Chilán et al. (2016) asegura que para garantizar la gestión efectiva del Gobierno Autónomo Descentralizado es primordial aplicar estrategias y procesos direccionado al logro de las metas, los mismos que deben ser medidos en base a la aplicación de los indicadores de eficiencia, eficacia y rendimiento, de modo que su aplicación pueda tener un impacto social positivo y se puedan ejecutar los proyectos de inversión comunales a favor del crecimiento de la localidad.

En Perú, Rojas (2015) sostiene que para garantizar la eficiencia de la ejecución de proyectos de inversión es primordial realizar un seguimiento efectivo de los procedimientos y acciones enfocadas a realizar las actividades de manera eficaz y eficiente basadas principalmente en las metas institucionales, las cuales buscan ejecutar programas, planes y proyectos a favor del desarrollo de la comunidad. Por su parte, la República del Perú (2018), dio a conocer a través de su balance anual, la existencia de problemas que afectaron la ejecución de proyectos, entre ellos las inconsistencias en los expedientes técnicos y procesos de mantenimiento prolongado, asimismo señalo que para optimizar la ejecución de los proyectos es importante intensificar procesos de fácil elaboración que erradiquen los obstáculos que afectan su desarrollo. Por otro lado, Mejía (2020), mencionó que la inversión pública a inicios del 2020, estaba entre S/ 1,023,000.00 millones, que representa un $89 \%$ más a diferencia del año anterior, por el contrario, pese a las cifras elevadas a inicios de año, las proyecciones de inversión bajaron los últimos meses (-8.5\%), por la paralización de obras, en el mes de mayo hubo una ejecución de S/ 6,3 mil millones, es decir solo un $13.4 \%$ de los S/ 47 mil que estuvieron en programación, que demuestra claramente que las entidades públicas poseen poca capacidad para enfrentar situaciones inesperadas, así como la falta de organización y funcionarios competentes para la ejecución de funciones. 
De igual manera, con la finalidad de brindar un soporte teórico a la investigación, se presentan los enfoques conceptuales concernientes a las variables. Bajo esa premisa, para proporcionar soporte bibliográfico a la variable eficiencia en la ejecución de proyectos de inversión, es esencial tener en cuenta lo dispuesto por el Decreto Legislativo $\mathrm{N}^{\circ} 1252$ (2020), donde menciona que comprende todas aquellas actividades, procesos y recursos públicos empleados para llevar a cabo una inversión con la finalidad de prestar servicios y provisionar infraestructuras para contribuir con el crecimiento del país.

En concordancia con la realidad problemática expuesta, se ha formulado como problema general ¿Cuáles son los principales hallazgos de eficiencia en la ejecución de proyectos de inversión pública en los últimos años?, planteando además como objetivo principal evaluar la eficiencia en la ejecución de proyectos de inversión pública en los últimos años.

\section{MÉTODOS}

La presente investigación tuvo un enfoque cualitativo por cuanto se revisó detalladamente los artículos científicos relacionadas con la eficiencia en la ejecución de proyectos de inversión, de modo que puedan ser utilizados como una herramienta informática que permita sintetizar toda la información concerniente al comportamiento de la variable dentro de diversos contextos (Rivadeneira, 2015).

Además, se ha considerado oportuno emplear un análisis sistemático debido a que la investigación estuvo enfocada a analizar de manera detallada los aspectos relacionados con la eficiencia en la ejecución de proyectos de inversión con la finalidad de identificar a través de las comparaciones realizadas las diferencias y similitudes que se presentan entre los diferentes contextos y reconocer los cambios significativos que se presentaron a lo largo de los años (Sánchez, 2019).

Por último, resulta indispensable mencionar también que la investigación presentó un diseño Descriptivo, debido a que buscó detallar la situación acerca del estado actual de las variables, describiendo de esa manera cada una de sus particularidades, características, cualidades y limitaciones, y sobre esa base llegar a plantear conclusiones certeras sobre la eficacia de la ejecución de proyectos a lo largo de los últimos años (Galeano, 2020). 


\section{RESULTADOS}

A continuación, se procede a efectuar un análisis de revisión, el mismo que será presentado de acuerdo a la siguiente tabla:

\begin{tabular}{|c|c|c|c|c|c|c|}
\hline $\mathbf{N}^{\circ}$ & Autor & País & Tipo y diseño & Muestra & Objetivos & Conclusiones \\
\hline 1 & $\begin{array}{ll}\text { Ruiz } & \text { y } \\
\text { Duarte } & \\
(2015) & \end{array}$ & Colombia & $\begin{array}{l}\text { Básica } \\
\text { Descriptiva }\end{array}$ & 34 entidades & $\begin{array}{l}\text { Evaluar la } \\
\text { ejecución de } \\
\text { los proyectos } \\
\text { de inversión }\end{array}$ & $\begin{array}{l}\text { La inversión pública en } \\
\text { los diversos proyectos de } \\
\text { desarrollo es de suma } \\
\text { relevancia e implican un } \\
\text { grado de importancia } \\
\text { alto para el desarrollo de } \\
\text { acciones de cada nación, } \\
\text { es decir, trata de } \\
\text { administrar el gasto } \\
\text { público de manera } \\
\text { eficiente, logrando que la } \\
\text { población quede } \\
\text { satisfecha ante sus } \\
\text { múltiples necesidades. }\end{array}$ \\
\hline 2 & $\begin{array}{l}\text { Ariza. } \\
(2017)\end{array}$ & Colombia & Mixta & $\begin{array}{l}230 \\
\text { instituciones }\end{array}$ & $\begin{array}{l}\text { Conocer la } \\
\text { efectividad } \\
\text { en el manejo } \\
\text { y control de } \\
\text { los proyectos } \\
\text { de inversión }\end{array}$ & $\begin{array}{l}\text { La eficiencia y eficacia } \\
\text { de la gestión de los } \\
\text { proyectos se fundamenta } \\
\text { por medio del aporte de } \\
\text { los proyectos a la } \\
\text { estrategia y la calidad de } \\
\text { gestión; asimismo, a } \\
\text { través de la aplicación } \\
\text { del modelo de } \\
\text { evaluación se pudo } \\
\text { conocer a once } \\
\text { indicadores entre los } \\
\text { cuales destaca el } \\
\text { cumplimiento de metas y } \\
\text { calidad de servicio. }\end{array}$ \\
\hline 3 & $\begin{array}{l}\text { Torrijos } \\
\text { (2016) }\end{array}$ & México & Explicativa & $\begin{array}{l}15 \text { proyectos de } \\
\text { inversión }\end{array}$ & $\begin{array}{l}\text { Evaluar el } \\
\text { diseño y } \\
\text { ejecución de } \\
\text { los proyectos } \\
\text { de inversión } \\
\text { local }\end{array}$ & $\begin{array}{l}\text { Para el diseño del } \\
\text { proyecto es primordial } \\
\text { considerar el ciclo de } \\
\text { vida del mismo y demás } \\
\text { aspectos relacionados a } \\
\text { ello como por ejemplo } \\
\text { los recursos materiales, } \\
\text { humanos, económicos y } \\
\text { financieros empleados. } \\
\text { Así pues, para la } \\
\text { ejecución resulta }\end{array}$ \\
\hline
\end{tabular}




\begin{tabular}{|c|c|c|c|c|c|c|}
\hline & & & & & & $\begin{array}{l}\text { relevante considerar los } \\
\text { indicadores de } \\
\text { transparencia } \\
\text { efectividad de la gestión } \\
\text { pública. }\end{array}$ \\
\hline 4 & $\begin{array}{l}\text { Candia } \\
(2015)\end{array}$ & Chile & Descriptiva & $\begin{array}{l}\text { Acervo } \\
\text { documentario } \\
\text { vinculado con } \\
\text { los proyectos } \\
\text { de inversión }\end{array}$ & $\begin{array}{l}\text { Identificar } \\
\text { las } \\
\text { deficiencias } \\
\text { que se } \\
\text { presentan en } \\
\text { la ejecución } \\
\text { de proyectos } \\
\text { de inversión }\end{array}$ & $\begin{array}{l}\text { Dentro de las principales } \\
\text { deficiencias evidenciadas } \\
\text { durante la ejecución de } \\
\text { los proyectos de } \\
\text { inversión destaca la falta } \\
\text { de control en el uso de } \\
\text { recursos públicos de tal } \\
\text { manera que se evalúe el } \\
\text { ingreso y salida de } \\
\text { recursos, la carencia de } \\
\text { mecanismos que evalúen } \\
\text { la capacidad competitiva } \\
\text { de los funcionarios } \\
\text { públicos. }\end{array}$ \\
\hline 5 & $\begin{array}{l}\text { Rebollar, } \\
\text { Posadas, } \\
\text { Rebollar, } \\
\text { Hernández } \\
\text { y Gonzáles } \\
(2020)\end{array}$ & Ecuador & Descriptivo & $\begin{array}{l}\text { Acervo } \\
\text { documentario } \\
\text { correspondiente } \\
\text { a la ejecución } \\
\text { de proyectos de } \\
\text { inversión }\end{array}$ & $\begin{array}{l}\text { Analizar a } \\
\text { inversión } \\
\text { privada en la } \\
\text { ejecución de } \\
\text { inversiones } \\
\text { públicas }\end{array}$ & $\begin{array}{l}\text { Se reconoce que la } \\
\text { inversión pública } \\
\text { destinada a la } \\
\text { planificación y ejecución } \\
\text { de los proyectos de } \\
\text { inversión es mínima, y } \\
\text { ello origina que no se } \\
\text { pueda disponer de los } \\
\text { recursos necesarios para } \\
\text { el desarrollo efectivo de } \\
\text { las actividades y } \\
\text { tampoco se efectúan } \\
\text { monitoreos de manera } \\
\text { continua con la finalidad } \\
\text { de evaluar la } \\
\text { competitividad de los }\end{array}$ \\
\hline
\end{tabular}




\begin{tabular}{|c|c|c|c|c|c|c|}
\hline & & & & & & funcionarios públicos. \\
\hline 6 & $\begin{array}{l}\text { Huaquisto } \\
\text { (2016) }\end{array}$ & Perú & $\begin{array}{l}\text { Correlacional } \\
\text { transeccional }\end{array}$ & $\begin{array}{l}8 \text { proyectos de } \\
\text { inversión } \\
\text { pública } \\
\text { ejecutados por } \\
\text { administración } \\
\text { directa }\end{array}$ & $\begin{array}{l}\text { Conocer la } \\
\text { relación que } \\
\text { existe entre } \\
\text { la eficiencia } \\
\text { y control del } \\
\text { proyecto con } \\
\text { el costo y } \\
\text { tiempo de } \\
\text { proyectos de } \\
\text { inversión }\end{array}$ & $\begin{array}{l}\text { El control y monitoreo } \\
\text { de la ejecución de } \\
\text { proyectos de inversión se } \\
\text { encuentra entre regular y } \\
\text { deficiente puesto que no } \\
\text { se cumplen de manera } \\
\text { adecuada las normas y } \\
\text { técnicas } \\
\text { correspondientes a la } \\
\text { ejecución de obras, } \\
\text { permitiendo deducir que } \\
\text { la eficiencia de tales } \\
\text { proyectos es menor al } \\
70 \% \text {. }\end{array}$ \\
\hline 7 & $\begin{array}{l}\text { Ferró } \\
\text { (2015) }\end{array}$ & Perú & $\begin{array}{l}\text { Descriptivo } \\
\text { Explicativo }\end{array}$ & $\begin{array}{l}10 \text { proyectos de } \\
\text { inversión }\end{array}$ & $\begin{array}{l}\text { Analizar la } \\
\text { intervención } \\
\text { de la } \\
\text { población en } \\
\text { el diseño y } \\
\text { ejecución } \\
\text { eficiente de } \\
\text { proyectos de } \\
\text { inversión }\end{array}$ & $\begin{array}{l}\text { La propuesta } \\
\begin{array}{l}\text { constituido } \\
\text { instrumento de } \\
\text { validez }\end{array} \text { en } \\
\text { administración de la } \\
\text { ejecución presupuestal } \\
\text { para los proyectos de } \\
\text { inversión del estado, } \\
\text { permitiendo un eficiente } \\
\text { uso de los recursos } \\
\text { públicos y garantizando } \\
\text { además la supervisión } \\
\text { oportuna de la misma. }\end{array}$ \\
\hline 8 & $\begin{array}{l}\text { Argüezo } \\
(2017)\end{array}$ & Perú & Básico & $\begin{array}{l}29 \text { proyectos de } \\
\text { inversión }\end{array}$ & $\begin{array}{l}\text { Evaluar la } \\
\text { eficiencia de } \\
\text { los proyectos } \\
\text { de inversión } \\
\text { pública }\end{array}$ & $\begin{array}{l}\text { El nivel de eficiencia } \\
\text { ejecución de proyectos } \\
\text { de inversión fue bajo, } \\
\text { por cuanto gran parte de } \\
\text { los proyectos } \\
\text { programados } \\
\text { correspondientes al }\end{array}$ \\
\hline
\end{tabular}




\begin{tabular}{|c|c|c|c|c|c|c|}
\hline & & & & & & $\begin{array}{l}\text { sector educación y salud } \\
\text { no fueron ejecutados } \\
\text { dentro de los plazos } \\
\text { señalados en un } 34 \% \text { y } \\
14 \% \text { respectivamente. }\end{array}$ \\
\hline 9 & $\begin{array}{l}\text { Magallanes } \\
(2016)\end{array}$ & Perú & $\begin{array}{l}\text { Aplicada } \\
\text { Explicativa }\end{array}$ & $\begin{array}{l}\text { Proyectos de } \\
\text { inversión de } 5 \\
\text { regiones }\end{array}$ & $\begin{array}{l}\text { Evaluar el } \\
\text { nivel de } \\
\text { eficiencia en } \\
\text { la ejecución } \\
\text { de proyectos } \\
\text { de inversión } \\
\text { públicos }\end{array}$ & $\begin{array}{l}\text { La eficiencia de la } \\
\text { ejecución de proyectos } \\
\text { tuvo un desempeño } \\
\text { medio en base a los } \\
\text { indicadores económicos } \\
\text { y sociales, por ello se } \\
\text { puede deducir que no } \\
\text { necesariamente la mayor } \\
\text { obtención de recursos } \\
\text { financieros implica una } \\
\text { mejora en el desarrollo } \\
\text { de los proyectos de } \\
\text { inversión. }\end{array}$ \\
\hline 10 & $\begin{array}{l}\text { Andía, } \\
\text { Velásquez y } \\
\text { Villena } \\
(2020)\end{array}$ & Perú & Descriptivo & $\begin{array}{l}25 \text { proyectos de } \\
\text { inversión } \\
\text { aprobados a } \\
\text { nivel regional y } \\
\text { local }\end{array}$ & $\begin{array}{l}\text { Analizar y } \\
\text { evaluar la } \\
\text { eficiencia de } \\
\text { los proyectos } \\
\text { de inversión } \\
\text { aprobados }\end{array}$ & $\begin{array}{l}\text { La calidad de la } \\
\text { eficiencia de los } \\
\text { proyectos de inversión } \\
\text { fue medio, puesto que ha } \\
\text { presentado una serie de } \\
\text { limitaciones } \\
\text { deficiencias a causa de la } \\
\text { falta de control y } \\
\text { evaluación del uso de los } \\
\text { recursos públicos y el } \\
\text { desempeño de los } \\
\text { funcionarios } \\
\text { competentes en base a } \\
\text { los indicadores } \\
\text { establecidos } \\
\text { (transparencia } \\
\text { economía). }\end{array}$ \\
\hline
\end{tabular}


La tabla presentada con anterioridad ha permitido conocer y analizar la manera en la que se presenta la eficiencia en la ejecución de proyectos de inversión a lo largo de los últimos años principalmente dentro de un contexto latinoamericano.

En tal sentido, estudios revisados sobre la eficiencia en la ejecución de proyectos de inversión permitieron identificar las diversas estrategias que aplican las organizaciones públicas con la finalidad de asegurar que los proyectos de inversión se ejecuten eficientemente, en donde se evidencia que la adopción de tales estrategias tienen un impacto significativo en los mismos por cuanto permiten que los proyectos se lleven a cabo dentro de los plazos programados con el propósito de contribuir con la consecución de los objetivos institucionales enfocados a mejorar de la calidad de vida de los pobladores y contribuir con el desarrollo del país. En tal sentido, se reconoce que entre las principales estrategias que comúnmente aplican las entidades e instituciones públicas destacan las siguientes: Planeación de las actividades, operaciones y procesos idóneos en concordancia con la misión, visión y planes institucionales de modo que se puedan comprometer a los recursos necesarios; organización de la ejecución de las actividades previamente planificadas de tal manera que puedan ser desarrolladas responsable y pertinentemente, asegurando así el cumplimiento de tales metas, del mismo modo es conveniente elaborar una guía o manual en donde se detallen minuciosamente los procesos a seguir y las acciones que se deben llevarse a cabo dentro de una entidad o institución al momento de realizar cada una de las actividades; seleccionar los recursos necesarios para ejecutar cada una de las actividades; desarrollar la capacidad de liderazgo de los jefes de las distintas áreas que comprenden una entidad o institución de tal manera que todos los integrantes puedan participar activamente en el planteamiento de medidas de corrección y mejora en pro del buen funcionamiento de la misma; monitorear y controlar continuamente los lineamientos, procesos y políticas aplicables en la entidad o institución de modo que su funcionamiento se encuentre alineado a las mismas para que se puedan cumplir los intereses colectivos, de igual manera controlar la realización de las labores por parte de los funcionarios y promover la motivación de manera constante con el propósito de optimizar sus capacidades y compromiso para con la entidad o institución de tal manera que pueda estar enfocado a una mejora continua, entre otros. Respecto a la realidad nacional, se reconoce que la adopción de estrategias dentro de las entidades e instituciones públicas es escasa, debido a que no se aplican durante el desarrollo de las actividades relacionadas con 
la ejecución de proyectos de inversión, lo cual suscita que el nivel de eficiencia de los proyectos de inversión sea bajo, por cuanto no son ejecutados de manera adecuada ni dentro de los plazos programados, además de producir malestar en los pobladores al no disponer de los bienes y servicios públicos requeridos. Por todo ello se puede percibir la necesidad de aplicar estrategias enfocadas a garantizar la eficiencia en la ejecución de proyectos de inversión, de tal manera que se pueda garantizar la consecución de los objetivos institucionales, siendo esto esencial para el crecimiento y desarrollo de la localidad y del país en general.

\section{DISCUSIÓN}

Mediante un análisis comparativo de los resultados presentados en un contexto internacional y nacional relacionados con la eficiencia en la ejecución de proyectos de inversión, se pudo recopilar la información precisa sobre la misma con el propósito de identificar semejanzas y diferencias con la presente investigación.

Así pues, los resultados de la investigación concernientes a la eficiencia en la ejecución de proyectos de inversión en un contexto internacional han permitido evidenciar que el nivel de eficiencia de los proyectos de inversión es bajo, por cuanto no son ejecutados de manera adecuada dentro de los plazos programados, lo cual produce malestar en los pobladores al no disponer de los bienes y servicios públicos requeridos. Frente a estos resultados es importante desatacar los resultados alcanzados por Ruiz, M y Duarte, T. (2015), pues ambos autores sostienen que la inversión pública representa una parte esencial para la pertinente ejecución de proyectos visto que coadyuva con la realización de actividades y obras públicas en beneficio de la sociedad, además de permitir la administración del gasto público de manera eficiente, logrando que los pobladores se sientas satisfechos frente a sus múltiples necesidades. De igual modo, Ariza, D. (2017) infiere que la efectividad de la gestión de los proyectos se fundamenta en la medida en la que los proyectos aporten con la calidad de gestión, frente a ello fue necesario la implementación de un modelo de evaluación basados en los indicadores de transparencia y economía de tal manera que se pueda asegurar la consecución de los objetivos y metas institucionales acordes con las necesidades de la población.

Por otro lado, en un contexto nacional, se evidencia la carencia de estrategias que contribuyan con la efectividad en la ejecución de proyectos de inversión, tales resultados se asemejan a los expuestos por Huaquisito (2016), donde se pudo evidenciar que la falta de 
control y monitoreo constante, así como la falta de cumplimiento de las normativas y procedimientos y la carencia de aptitudes necesarias por parte de los funcionarios competentes, suscitó que alcance un bajo nivel de eficiencia pues los proyectos de inversión no son ejecutados dentro del tiempo establecido y con los recursos económicos consignados. Por el contrario, estos resultados no son similares a los presentados por Ferró (2015), visto que el autor aseveró que la implementación de una propuesta ha contribuido de manera significativa originando que los proyectos de inversión sean ejecutados en los plazos programados y con los recursos públicos determinados inicialmente, sin necesidad de solicitar adicionales.

Por todo ello se puede percibir la necesidad de proponer estrategias enfocadas a garantizar la eficiencia en la ejecución de proyectos de inversión, de tal manera que se pueda garantizar la consecución de los objetivos institucionales, siendo esto esencial para el crecimiento y desarrollo de la localidad y del país en general.

\section{CONCLUSIONES}

Por medio de la revisión a las investigaciones afines al presente artículo se pudo percibir la existencia de diversos inconvenientes y deficiencias concernientes a la eficiencia en la ejecución de proyectos de inversión. En tal sentido, se considera oportuno mencionar la importancia que tienen las estrategias de gestión pública frente a tales deficiencias debido a que comprenden una serie de acciones a través de las cuales se puede optimizar el funcionamiento de una entidad pública con la finalidad de asegurar el cumplimiento de sus planes y proyectos programados, los mismos que fueron diseñados en base a los requerimientos y necesidades prioritarias de los pobladores, y contribuir con la ejecución oportuna de las obras y proyectos de inversión de modo pertinente. En tal sentido, para asegurar la eficiencia en la ejecución de proyectos de inversión resulta sumamente necesario adoptar estrategias de gestión acordes con las necesidades de la entidad e institución pública con la finalidad de identificar oportunamente las deficiencias que afectan su correcta ejecución y determinar medidas correctivas frente a las mismas para su pronta solución.

Por tal motivo, se reconoce la necesidad de las entidades e instituciones públicas de implementación de medidas estratégicas de gestión pertinentes que permitan asegurar la eficiencia en la ejecución de proyectos de inversión de tal manera que los recursos públicos 
puedan ser empleados adecuadamente y las actividades se realicen cabalmente en función a lo programado.

\section{REFERENCIAS}

Andía, W., Velásquez, J. y Villena, R. (2020). La evaluación de proyectos de inversión en el sector saneamiento del Perú: Análisis metodológico. Dominio de las Ciencias. 6(3), 225-241. https://dialnet.unirioja.es/descarga/articulo/7562484.pdf

Argüezo, A. (2017). El presupuesto participativo en los proyectos de inversión pública en el Gobierno Regional Huánuco, 2012-2014. Revista Gaceta Científica. 3(2), 119124. http://revistas.unheval.edu.pe/index.php/gacien/article/view/409

Ariza, D. (2017). Efectividad de la gestión de los proyectos: una perspectiva $\begin{array}{lllll}\text { constructivista. } & \text { Obras } & \text { y }\end{array}$ https://scielo.conicyt.cl/pdf/oyp/n22/0718-2805-oyp-22-0075.pdf

Candia, R. (2015). Ideas para mejorar procesos de inversión pública como forma de reducir disparidades territoriales del desarrollo. Revista Estudios de Políticas Públicas. 1(2), 226-231. https://dialnet.unirioja.es/descarga/articulo/6067301.pdf

Chilán, S. et al. (2016). Modelo de gestión administrativa para optimizar el desempeño de los Gobiernos Autónomos Descentralizados. Investigación y pensamiento crítico. 5(2), 99-113. https://dialnet.unirioja.es/servlet/articulo?codigo=5503960

Decreto Legislativo $\mathrm{N}^{\circ}$ 1252. Decreto Legislativo que crea el Sistema Nacional de Programación Multianual y Gestión de Inversiones. Diario Oficial El Peruano. Perú. https://www.mef.gob.pe/es/normatividad-inv-publica/instrumento/decretoslegislativos/15603-decreto-legislativo-n-1252/file

Ferró, P. (2015). Participación de la población en la elaboración de proyectos de inversión pública: Un análisis según el grado de ruralidad para las provincias de Puno y el Collao, 2012 Perú. Revista de Investigación en Comunicación y Desarrollo. 6(1), 16-27. https://www.redalyc.org/pdf/4498/449844869002.pdf

Galeano, M. (2020). Diseño de proyectos en la investigación cualitativa. Colombia: Editorial de la Universidad EAFIT

Gobierno Regional de San Martín. (2019). Proyectos de inversión Pública. http://www.pehcbm.gob.pe/estudios/ 
Gonzáles, J. (2019). Flexibilizar la gestión administrativa del Estado colombiano en tiempos de globalización. Estudios de Derecho. 76(168), 43-71. https://doi.org/10.17533/udea.esde.v76n168a02

Huaquisto, S. (2016). Análisis de eficiencia en proyectos de inversión pública: Un estudio de caso en proyectos ejecutados por administración directa. Revista de Investigación Altoandina. $\quad 18(1), \quad 61-68$. https://dialnet.unirioja.es/servlet/articulo?codigo=5399051

Magallanes, J. (2016). Eficiencia económica de la inversión pública financiada con recursos del canon y regalías mineras en el Perú. Revista Anales Científico. 77(2), 309-318. https://dialnet.unirioja.es/descarga/articulo/6171213.pdf

Mejía, M. (2020). Inversión pública de enero logra crecimiento histórico. Revista Andina. https://andina.pe/agencia/noticia-mef-inversion-publica-enero-logra-crecimientohistorico-783628.aspx

Rebollar, S., Posadas, R., Rebollar, E., Hernández, J. y Gonzáles, F. (2020). Aportes a indicadores de evaluación privada de proyectos de inversión. Estudios de Economía. 44(2), 131-156. https://scielo.conicyt.cl/pdf/ede/v44n2/0718-5286-ede44-02-00131.pdf

República del Perú. (2018). Informe de actualización de proyecciones macroeconómicas 2018 - 2021. Reporte del Ministerio de Economía y Finanzas. https://www.mef.gob.pe/contenidos/pol_econ/marco_macro/IAPM_2018_2021.pdf

Rivadeneira, E. (2015). Comprensión teórica y proceso metodológico de la Investigación $\begin{array}{llll}\text { Cualitativa. In } & \text { Crescendo Institucional. }\end{array}$ https://revistas.uladech.edu.pe/index.php/increscendo/article/view/1179/926

Rojas, P. (2015). Public Administration and the Principles of Administrative Law in Peru. Revista Digital de Derecho Administrativo. 1(13), 193-209

Ruiz, M. y Duarte, T. (2015). Los proyectos de desarrollo: la inversión pública y la inversión privada. Revista Scientia Et Technica. 20(2), 135-137. https://www.redalyc.org/pdf/849/84942286007.pdf

Sánchez, F. (2019). Fundamentos Epistémicos de la Investigación Cualitativa y Cuantitativa: Consensos y Disensos. Revista Digital de Investigación en Docencia Universitaria. 13(1), 102-122. https://doi.org/10.19083/ridu.2019.644 
Torrijos, A. (2016). Propuesta metodológica en proyectos públicos de inversión real bajo el principio de desarrollo sostenible. Revista de Ciencias Sociales y Humanidades. 25(50), 23-48. http://dx.doi.org/10.20983/noesis.2016.2.2 